\title{
Linear models of dissipation whose $Q$ is almost frequency independent $(*)$
}

\author{
M. CAPUto (**) \\ Riceruto il 31 Maggio 1966
}

Summary. --. Laboratory experinents and fied observations indicate that the $Q$ of many non ferromannetiv inorganic solids is almost frequency independent in the range $10^{7}$ to $10^{-2}$ eps; althongh no single substance has been investigated over the entire frequency spectrun. One of the purposes of this investigation is to find the analytic expression of a linear dissipative mechanism whose $Q$ is alnust frequency independent over large frequency ranges. This will be obtaines by introlueing fractional derivatives in the stress strain relation.

Since the aim of this restarch is to also contribute to elueidating the dissipating mechanisin in the earth free modes. we shall treat the eases of dissipation in the free purely torsinnal modes of a shell and the purely railial vibration of a solid iphere.

The theory is elecelsell with llye mew valuen determined for the of of the spleroilat tree monles of the eath in the range between 10 and 5 minutes integrated with the $Q$ of the Railegh waves in the range between 5 and 0.6 minutes.

Another check of the theory is unde with the experimental values of the $Q$ of the longitulinal waves in an alluminum rod, in the range between $10^{-5}$ and $10^{-3}$ seconds.

In both cheks the theory represents the observed phenomena very satisfactorly.

Rassunto. ... I risultati delle: ricerehe di laboratorio e delle osservazioni in fenomeni naturali inslicano che il $Q$ di parechi solidi inorganic: non ferromagnetici ì indipendente dalle frequenze nell'intervallo $10^{-2}$, $10^{7}$ cicli al secondo; per quanto nessunia sostanza sia stata studiata in tutto

$\left.{ }^{*}\right)$ This paper was presented at the 1966 ammual meeting of $\mathrm{AGL}^{\top}$ in Washington I)C.

(**) Department of Geoplysics, Liniversity of British C'olumbia, Canada. 
questo intervallo di frequenze. Cho degli seopi della presente ricerca è quello di trovare l'espressione analitica di un modello di dissipazione lineare in eui $Q$ sia indipendente dalla frequenza in un vasto intervallo di frequenze. Questo sarà ottenuto introducendo derivate di ordine frazionario nelle relazioni fra sforzo c deformazione.

Poiché uno degli scopi di questa ricerea è anche di contribuire ad una miglior comprensione tei mectanismi di dissipazione dell'energia nelle oscillazioni libere della Terra, in questa nota si applicherà la legge di dissipazione citata al caso delle oscillazioni torsimali libere di uno strato sferico.

La teoria espostat riene poi applicala allo studio dei valori di $Q$ osservati nelle onde di Rayleight e nelle oscillazioni sferoidali della Terra.

Un'altra applienzione della teoria e fatta allo studio lei valori di $Q$ osservati nelie onkle longitudinizli di unit sbarra di alluminio.

In entrimbe le applicizioni la teoria rappresenta in maniera soddisfacente i fenomeni osservati.

\section{IXTRODUCTION}

In a homogeneous isotropic elastic field the elastic properties of the substance are specified by a description of the strain and stre sses in a limited portion of the field since the stratins and stresses are linearly related by two paraneters which describe the elastic properties of the field. If the elastic field is not homogeneous nor isotropie the properties of the field are specified in a similar manner by a larget number of parmaters which also rlepend on the position.

These perfectly alastic fields are insufficient models for the description of malny physical phemomena beratuse they do not allow to explain the dissipation of mergy. A more complete deseription of the actual elastic fields is obtained by introduring stress-strain lelations which include also linear combinations of time derivatives of the strain and the stress. The numerical cocficients appearing in the general linear combinations of higher order derivatives are alled viseoelastic constants of higher onder.

IHastic ficlds described by clastir constants of higher order have been discussed by many author's, [( Knopoff studied the case in which the stress strain relations are of the type

$$
\tau_{r s}=\lambda g^{h i} g_{r i} e_{h i}+2 \mu e_{r s}+\frac{d^{m}}{d \overline{t^{m}}}\left(\lambda_{m} g^{h i} g_{r s} e_{h i}+2 \mu_{m t} c_{r s}\right)
$$

where $\lambda_{m}$ and $\mu_{m}$ are constant, he obtained a condition for these viscoelastic constants of higher order analogous to those existing for the 
perfectly elastic fiolds and also jroved that in order to have a dissipative elastic field the stress-strain relations should contain time derivatives of odd order.

A generalization of the relation [1] is

$$
\tau_{r s}=\sum_{0}^{p} \frac{d^{m}}{d t^{m}}\left[\lambda_{m} g^{h i} g_{r s} e_{h i}+-2 \mu_{m} e_{r s}\right]
$$

where one can also consider $\hat{i}_{m}$ and $\mu_{m}$ functions of position.

We can generaijo [2] to the ese when the operation $\frac{d^{m}}{d t^{m}}$ is perforened with $m$ as a real number $z$ (see appendix) and also further by substituting the summation with an integral as follows

$$
\tau_{r s}=\int_{a_{1}}^{b_{1}} f_{1}(r, z) \frac{d^{z}}{d t^{z}} g^{h i} g_{r s} e_{h i} d z+2 \int_{a_{2}^{\prime}}^{b_{2}} f_{2}(r, z)-\frac{d z}{d t^{z}} e_{r s} d z
$$

$\mu$ is the radial coordinate in a spherical coordinate system.

Relations [1] and [2] are a special case of [3] - they are obtained by setting

$$
\begin{aligned}
& f_{1}(r, z)=\sum_{q}^{p} \delta(z-m) \lambda_{m} \\
& f_{2}(r, z)=\sum_{q}^{p} \delta(z-m) \mu_{m}
\end{aligned}
$$

where $\delta(z-m)$ are unitaly delta functions.

If $a_{i}=q=p=0$, then we have the ense of a purfectly elastic field: if $q=p=1$ than we have a perfectly viscous field; if $q=a_{i}=0$ and $p=1$ then we have a viscoclastice field.

Dissipation in a planc wave

In the simple case of a plane wava, assuming $f:=\eta \delta\left(z-z_{0}\right)$, the stress strain relation [3] gives the following equation of motion

$$
\varrho \frac{\partial^{2} u}{\partial t^{2}}+\mu \frac{\partial^{2} u}{\partial x^{2}}+\eta \frac{\partial^{z_{0}}}{\partial t^{z_{0}}} \frac{\partial^{2} u}{\partial x^{2}}=0 .
$$

By taking the Taplace transform of [5] we have

$$
\varrho p^{2} U+\mu \frac{\partial^{2} U}{\partial x^{2}}+\eta p^{z_{0}} \frac{\partial^{2}}{\partial x^{2}}=0
$$


and the nature of the motion depends on the roots of the following equation

$$
\eta \alpha^{2} p^{z_{o}}+\varrho p^{2}+\mu \alpha^{2}=0 .
$$

The approximate solution of [6], neglecting the term in $\eta$, which we assume to be small with respect to $\mu$, is

$$
p^{2}=-\frac{\mu \alpha^{2}}{\varrho}
$$

the solution which takes into account the dissipation is

$$
p=i\left|p_{o}\right| 1+\left\{\frac{\eta\left|p_{0}\right|^{z_{o}} a^{2}}{2\left|p_{o}\right|^{2} \varrho} \quad\left(\cos \frac{\pi}{2} z_{0}+i \sin \frac{\pi}{2} z_{0}\right)\right\}
$$

and the specific dissipation is

$$
Q^{-1}=-\frac{\eta\left|p_{o}\right|^{z_{o}}}{\mu} \cdot \sin \frac{\pi}{2} z_{o}
$$

Solution of the equations of motion in spherical coordinates

We shall follow the method described in Caputo [1966]; the operatior o, introduced in that paprer is

$$
o_{t}=\nu_{i j}(r) \frac{\partial j}{\partial t^{j}}
$$

here, accolding to the defintion [3] of the stress-strain relation, these operators will be

$$
\begin{aligned}
& o_{i}=\int_{a_{1}}^{b_{1}} f_{1}(r, z) \frac{\partial z}{\partial t^{z}} d z-f-2 \int_{a_{1}}^{b_{1}} f_{2}(r z) \frac{\partial^{z}}{\partial t^{z}} d z \\
& o_{2}=\int_{a_{1}}^{b_{1}} f_{2}(r, z) \frac{\partial^{z}}{\partial t^{z}} d z .
\end{aligned}
$$

One can sec that the method of solving the equations of equilibrium resulting from the definition [1] of the stress-strain relation (see Caputo 1966) can be applied also to the ease when the estress-strain relation is [3]. 
The Laplace tiansform $s\left(S_{r}, S_{\vartheta}, S_{\varphi}\right)$ of the displacement vector $s\left(s_{r}, s_{\vartheta}, s_{\varphi}\right)$ - where $r, \eta, \varphi$ are spherical coordinates, 9 colatitude, $\varphi$ longitude - is:

$$
\begin{aligned}
& S_{\vartheta}=\sum_{n} \sum_{k} R_{1} Y_{n}^{k} \\
& S_{\vartheta}=\sum_{n} \sum_{k}\left(R_{3} \frac{\partial Y_{n}^{k}}{\partial \vartheta}: R_{3} \frac{1}{\sin \vartheta} \frac{\partial Y_{n}^{k}}{\partial \varphi}\right) \\
& S_{\varphi}=\sum_{n} \sum_{k}\left(R_{2} \frac{1}{\sin \vartheta} \cdot \frac{\partial Y_{n}^{k}}{\partial \varphi}-R_{3}-\frac{\partial}{\partial \vartheta}\right)
\end{aligned}
$$

where $Y_{n}^{k}$ are spherical harmonics

$$
Y_{n}^{k}= \begin{cases}\left(\frac{2 n+1}{4}\right)^{1 / 2} P_{n}(\cos \vartheta) & \text { if } k=0 \\ \left(\frac{2 n+1}{2} \frac{(n-K) !}{\left(n+\frac{K}{K}\right)}\right)^{1 / 2} P_{n}^{(k)}(\cos \vartheta) \cos K \varphi & \text { if } k=1,2, \ldots, n[12] \\ \left(\frac{2 n+1}{2} \frac{(2 n-K) !}{K !}\right)^{1 / 2} P_{n}^{(k-n)}(\cos \vartheta\} \cos (k-n) \varphi, \text { if } k=n+1, \ldots, 2 n\end{cases}
$$

and $R_{1, n}, R_{2, n}, R_{3, n}$ are solutious of the system

$$
\begin{aligned}
& \left.\frac{d}{d r}\left[O_{1} \overline{\bar{\nabla}}\right]+2 O_{2} \frac{d \bar{\nabla}}{d r}-O_{2}{ }^{\prime} R_{1} \cdot n(n+1)-\frac{1}{r^{2}} \frac{d\left(r R_{2}\right)}{d r} n(n+1)\right]+ \\
& 2 \dot{O}_{1} \frac{d R_{1}}{d r}-\varrho \frac{d P_{o}}{d r} \nabla=\frac{d\left(P-P_{o}\right)}{d r}+\varrho \frac{d}{d r}\left(R_{1} \frac{d P_{o}}{d r}\right)-p^{2} \varrho R_{1}=0
\end{aligned}
$$

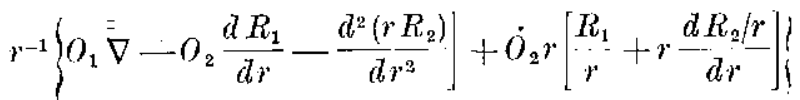

$$
\begin{aligned}
& +\frac{d}{d r}\left(P-P_{0}\right)+\varrho \frac{R_{1}}{r} \frac{d P_{o}}{d r}-p^{2} \varrho R_{2}=0 \\
& r^{-2} \frac{d}{d r}\left[r^{2} \frac{d\left(P-P_{0}\right)}{d r}\right]-4 \pi \varrho G \bar{\nabla} \nabla+\frac{n(n+1)}{r} \frac{P-P_{o}}{r}-4 \pi G \varrho \frac{d R_{1}}{d r}=0 \\
& O_{2}\left[\frac{1}{r} \frac{d^{2} r R_{2}}{d r^{2}}-\frac{n(n+1)}{r^{2}} R_{3}\right]+\dot{O}_{2} r \frac{d R_{3} / r}{d r}+O_{3} R_{3}=0 \\
& \overline{\bar{\nabla}}=-\frac{n(n+1)}{r} R_{2}+\frac{1}{r^{2}} \cdot \frac{d}{d r}\left(r^{2} R_{1}\right)
\end{aligned}
$$

with

$$
P-P_{0}=\int_{0}^{2 \pi} \int_{0}^{\pi} \int_{0}^{\infty}\left(V-V_{0}\right) e^{-p t} \sin \vartheta d \varphi d \vartheta d t .
$$


$V-V_{0}$ is the perturbation of the gravitational potential arising from the perturbation of the density field and from the attraction of the density perturbation at the deformed interfaces.

We assume also that the integration in [3] can be interchanged with the integration of the Laplace transformation and also that we can use formula [38] (see appendix at the end of the paper). Then e.g.

$$
O_{2}=\int_{i}^{\infty} o_{2} s_{1} e^{-p t} d t=\left(\left|\int_{i_{2}}^{b_{2}} p^{z} f_{2}(r, z) d z\right| \int_{0}^{\infty} s_{i} e^{-p t} d t\right.
$$

Dissipation in the torsional oscillation of a shell

We shall discuss some models of dissipation, obtained by specializing the functions $f_{i}(r, z)$ apperaring in [3], in the free purely torsional and radial vibrations of a shell.

The equation which governs the motions of the torsional modes in a non perfectly elastic shell of radiat $r_{1}$ and $r_{2}$, assuming a stress-strain relation of the type [3], is:

$$
\begin{gathered}
\int_{a_{2}}^{b_{2}} f_{2}(r, z) \frac{d^{z}}{d t^{2}}\left|\frac{1}{r} \frac{\partial^{2} r s}{\partial r^{2}}+\frac{1}{r} \frac{\partial}{\partial \vartheta}\left(\frac{1}{\sin } \frac{\partial s \sin \frac{\vartheta}{\partial \vartheta}}{\partial \theta}\right)\right| d z+ \\
+\int_{a_{2}}^{b_{2}}\left(\frac{\partial}{\partial r} f_{2}(r, z)\left|\frac{d^{z}}{d t^{2}}\right| r \frac{\partial}{\partial r}\left(\frac{s}{r}\right)\right\rfloor d z=\varrho \frac{\partial^{2}}{\partial} \frac{s}{t^{2}}
\end{gathered}
$$

We assume an Earth model defined by a liquid core and a homogeneous mantle, and assume also that the dissipation of energy due to the viscous interaction between the core and the mantle, is negligible [Caputo, 1966] and that $\frac{\partial}{\partial r} f_{2}(r, z)=0$, then the boundary condition is

$$
\begin{array}{lr}
F_{n}\left(r_{2}\right) & F_{-n}\left(r_{2}\right) \\
F_{n}\left(r_{1}\right) & F_{-n}\left(r_{1}\right) \\
F_{ \pm n}(r)=\frac{d}{d r} r^{-3 / 2} J(r \alpha) \\
\pm n+1 / 2
\end{array}
$$


where

$$
\begin{gathered}
S_{\mathrm{3}}=\left\{A_{n} J_{n+1 / 2}(a r)+(-1)^{n+1} A_{-n} J_{-n-1 / 2}(a r)\right\} r^{-1 / 2} \frac{d P_{n}}{d \vartheta} \\
\alpha^{-2}=\varrho_{\dot{b_{2}}}^{-1}{\dot{\dot{a}_{2}}}_{f_{2}(z) p^{z} d z}^{a_{2}}
\end{gathered}
$$

is a solution of the Isaplace transform of [15]. $J_{-n-1 / 2}(\alpha r)$ and $J_{n+1 / 2}(\alpha r)$ are Bessel functions, and $P_{n}(\cos \vartheta)$ are Legendre polinomials.

The solutions of [16] determine the periods of free oscillation and also the $Q$. Without loss of generality $[10]$ can be written

$$
f_{2}(z)=\mu+\int_{a_{2}}^{b_{2}} \overline{f_{2}}(z) p^{2} d z
$$

An interesting case arises when

$$
\overline{f_{2}}(z)=\mu_{1} \delta\left(z-z_{o}+\varepsilon\right)
$$

equation [1.6] is then

$$
-\frac{\alpha^{2}}{\varrho}\left|\mu+\mu_{1} p^{z_{0}-\varepsilon}\right|=p^{2}
$$

which gives

$$
p=i\left|p_{o}\right|\left\{1+\frac{\mu_{1}}{\mu} p_{o}^{z_{o}-\varepsilon}\right\}^{1 / 2}, \quad\left|p_{o}\right|=\alpha \sqrt{\frac{\mu}{\varrho}}
$$

If $z_{o}=2 m$ ( $m$ integer) then we have

$$
p=i\left|p_{\circ}\right|\left\{1+\frac{\mu_{1}\left|p_{o}\right|^{2 m-\varepsilon}}{\mu}(-1)^{m}\left[\cos \frac{\pi \varepsilon}{2}-i \sin \frac{\pi \varepsilon}{2}\right]\right\}^{1 / 2}
$$

and if $m=0$

$$
p=i\left|p_{o}\right|\left\{1+\frac{\mu_{1}\left|p_{o}\right|^{-\varepsilon}}{2 \mu}\left[\cos \frac{\pi \varepsilon}{2}-i \sin \frac{\pi \varepsilon}{2}\right]\right\}
$$

The specific dissipation function is therefore

$$
Q^{-1}=\frac{\mu_{1}}{\mu}\left|p_{o}\right|^{-\varepsilon}\left|\sin \frac{\pi}{2} \varepsilon\right|
$$


Dissipation in the purely radial modes

The study of the dissipation of energy in the purely radial modes, can be done as that of the torsional modes. The only difference is that in this case the forces which grovern the periods of the modes are the elastic forees and also the gravitational forces. Assuming a homogeneous earth model of density $\varrho_{0}$ and $\frac{\partial f_{1}}{\partial r}=0$ one can see that the periods of the free modes and the dissipation are given by

$$
\begin{aligned}
& \varrho_{o} r_{2}^{2}\left(p^{2}-4 A\right)=-x^{2} f_{a_{1}}^{b_{1}}(z) p^{z} d z \\
& A=\frac{4 \pi}{3} \underline{G} \varrho_{o}
\end{aligned}
$$

and $x$ is a solution of

$$
\frac{\tan x}{x}=\frac{1}{\left.1-\frac{x^{2}}{4} 2+\frac{\lambda}{\mu}\right)} .
$$

Without loss of generality $f_{1}$ can be written

$$
f_{1}=\lambda+2 \mu+\int_{a_{1}}^{b_{1}} \overline{f_{1}}(z) p^{z} d z .
$$

An interesting case arisen when

$$
\bar{f}_{1}(z)=\lambda_{1} \delta\left(z-z_{0}+\varepsilon\right)
$$

equation $[25]$ is then

$$
\begin{aligned}
p^{2} & =4 A-\frac{x^{2}}{\varrho r_{2}^{2}}\left(\lambda+2 \mu+\lambda_{1} p^{z_{0}-\varepsilon}\right)=-\left|p_{0}\right|^{2}-\frac{x^{2} \lambda_{1}}{\varrho r^{2}} p_{o}^{z_{0}-\varepsilon} \\
& =-\left|p_{0}\right|^{2}-\left\lfloor\frac{x^{2}}{\varrho r_{2}^{2}}(\lambda+2 \mu)-4 A\right] \frac{\lambda_{1} p_{0}^{z_{0}-\varepsilon}}{\lambda+2 \mu}+\frac{4 A \lambda_{1}}{\lambda+2 \mu} p_{o}^{z_{0}-\varepsilon} \\
& p_{o}^{2}=4 A-\frac{x^{2}}{\varrho r_{2}^{2}}(\lambda+2 \mu) .
\end{aligned}
$$


Its solutions are

$$
p= \pm i\left|p_{0}\right|\left\{1+\frac{\lambda_{1}}{\lambda+2 \mu} p^{z_{o}-\varepsilon}-\frac{4 A \lambda_{1} p_{o} z_{o}-\varepsilon}{(\lambda+2 \mu)\left|p_{o}\right|^{2}}\right\}^{1 / 2}
$$

which becomes for $z_{o}=0$

$$
p= \pm i\left|p_{o}\right|\left\{1+\frac{\lambda_{1}}{\lambda+2 \mu} p_{o}^{-\varepsilon}-\frac{4 A \lambda_{1} p_{0}^{-\varepsilon}}{(\lambda+2 \mu)\left|p_{o}\right|^{2}}\right\}^{1 / 2} .
$$

Equation [31] can also be written

$$
p= \pm i\left|p_{o}\right|\left\{1+\frac{\lambda_{1}\left|p_{o}\right|^{-\varepsilon}}{2\left(\lambda_{0}+2 \mu\right)}\left\{1-\frac{4 A}{\left|p_{o}\right|^{2} \mid} \mid\left[\cos \frac{\pi}{2} \varepsilon-i \sin \frac{\pi}{2} \varepsilon\right]\right\}\right.
$$

and the specific dissipation is

$$
Q^{-1}=\frac{\lambda_{1}\left|p_{0}\right|^{-\varepsilon}}{\lambda+2 \mu}\left|\sin \frac{\pi}{2} \varepsilon\right|\left\{1-\frac{4 A}{\left|p_{0}\right|^{2}}\right\}
$$

The ratio of the observed $Q$ 's gives $\varepsilon$

$$
\frac{Q_{0}^{-1}}{Q_{1}^{-1}}=\left(\frac{\left|p_{0 o}\right|}{\left|p_{0_{1}}\right|}\right)^{-\varepsilon}, \quad \varepsilon \ln \frac{\left|p_{o o}\right|}{\left|p_{o_{1}}\right|}=\ln \frac{Q_{0}}{Q_{1}},
$$

$\lambda_{1}$ is therefore obtained from [34] by substitution

$$
\lambda_{1}=\frac{(\lambda+2 \mu)\left|p_{0}\right|^{\varepsilon}}{Q\left|\sin \frac{\varepsilon \pi}{2}\right|} \text {. }
$$

\section{Ohecks with the observations}

A very extensive antysis of the attenuation of the Rayleigh waves has been made by Ben-Menahem (1964) who measured it from four great earthquakes from observation of multiple circuits around the earth past one station. By Fourier analysis be obtained the specific attenuation factor in the period range between 300 and 40 seconds; from the attenuation factor he computed then the specific dissipation.

The discrete spectium of the spheroidal oscillations of the earth, where the matter is both compressed and sheared, approaches the continuous Rayleigh waves spectrum. In order to extend the range where the $Q$ is known, we computed it also from the fres spheroidal oseillations 
of the Earth of period between so and 18 minutes, using the 110 hours record fo the 1961 Chilean quake recorded at UCLA. The record was subdivided in several intervals whose initial points where 4.5 and 13.5 hours apart; from the power spectral analysis of these intervals we obtained the decrease in enery and subsequently the $Q$ 's by means of formula [24] of Caputo (1966). From a meliminary analysis of these $Q$ 's we can see that the agrecment with the mathematical model of dissipation proposed in this papere, with $\varepsilon--0.75$, is satisfactory.

Another sheck of this theory "an be mule using the $Q$ 's obtained by Zemaneck and Rudmik (1961) for longitudinal wares in the period range between $10^{-3}$ and $10^{-5}$ seconds. Hexe again the agreement of the observed Q's with the model of dissipation proposed in the present paper, with $\varepsilon=-0.15$, is satisfartory. We plan to complete and publish soon the discussion of the above montioned experimental results.

\section{Appendix}

A generalization of the operation of differentiation with real order of differentiation, for a wide class of analytic functions, an be made as follows.

Let

$$
\frac{d^{z}}{d t^{z}}\left(c t^{x}\right)=\left\{\begin{array}{ll}
e \frac{\Gamma(x+1)}{\Gamma(x+1-z)} t^{x-z} & \text { if } x>0 \\
0 & \text { if } x=0
\end{array}\right\} \begin{aligned}
& 0<z<1 \\
& x \text { integer }
\end{aligned}
$$

It $f(t)$ is an analytic function, the operator [36] can be applied to the terms of its power series expansion; the resulting series, if convergent, can be assumed to be the $z$ order rerivative of $f(t)$.

We shall need to evaluate the Laplace transform of derivatives of order $z$ of a class of analytic functions. Let the power series expansion of $f(t)$, convergent in the interval $(0, \infty)$, be

$$
\begin{gathered}
f(t)=\sum_{i} a_{i} t^{i} \\
a_{i}=0 \quad \text { if } i \leqslant 0
\end{gathered}
$$

and, differentiating,

$$
\frac{d^{z} f(t)}{d t^{z}}=\Sigma_{i} a_{i} \frac{\Gamma(i+1)}{\Gamma(i+1-z)} t^{i-z} .
$$


We want to prove that for $0<z<1$

$$
\int_{0}^{\infty} \frac{d z f(t)}{d t^{2}}-e^{-p t} d t=p^{2} f(t) e^{-p t} d t
$$

To obtain [38] we shall assume that the Laplace transform of $\frac{d^{z} f(t)}{d t^{2}}$ exists, than we have

$$
\int_{i}^{\infty} \frac{d^{z} f(t)}{d t^{2}} t^{-p t} d t=\int_{0}^{\infty} \sum_{i} \frac{\Gamma(i+1)}{\Gamma^{\top}(i+1-z)} \cdot t^{i-2} t^{-p t} d t
$$

and, assuming that it is possible to interchange the sum with the integral

$$
\begin{gathered}
\sum_{i} a_{1} \frac{\Gamma(i+1)}{\Gamma(i+1-z)} \int_{0}^{\infty} t^{i-z} e^{-p t} d t=\sum a_{i} \Gamma(i+1) p^{2-1-i}= \\
=p^{z} \sum_{i} a_{i} \Gamma(i+1) p^{-p-1}=p^{z} \sum a_{l} \int_{t^{i} e^{-p t} d t=}^{\infty} \\
=p^{z} \int_{0^{\infty}}^{\infty} \sum a_{i} t^{i} e^{-p t} d t=p^{z} \int_{i}^{\infty} f(t) e^{-p t} d t
\end{gathered}
$$

which proves [38].

\section{RLPERFNCES}

Bex-Menafym A., Allemuation of seismic surface wates in the upper mantle 1964.

Caruto M., Estimates of anelastic dissipalion in the Earth's torsional modes. "Annali di Geofisica", 1, 75-94, (1966).

Kropork L., On the dissipative eiscoelastic constants of higer order. "J. Acoustic Soc. Am.", 26, 183.186, 1954.

ZEMANEK J. Jr., RuDNick 1., Attenuation and dispersion of elastic waves in a eylindrical bar. "J. Acoust. Soc. Ant.", 33, 1283-1288, (1961). 